\title{
EXCLUSIVIDAD Y FRAGMENTACIÓN: LOS PERFILES CULTURALES DE LA CLASE MEDIA EN CHILE ${ }^{1}$
}

\author{
Exclusivity and fragmentation: \\ cultural middle class-ness in Chile
}

\author{
Modesto Gayo $(*)$ \\ Berta Teitelboim (**) \\ María Luisa Méndez $\left.{ }^{(* *}\right)$
}

\begin{abstract}
RESUMEN
La literatura sociológica sobre la clase media alta, desarrollada fundamentalmente en la segunda parte del siglo XX, nos ha permitido conocer en detalle su comportamiento político y cultural, principalmente en los casos de Europa occidental y Estados Unidos. De este trabajo podemos extraer dos tesis que configuran la imagen de esta clase social: la exclusividad y la fragmentación. Exclusividad porque sus miembros muestran un comportamiento político frecuentemente similar al de las clases más altas de la sociedad y un patrón cultural en donde el refinamiento y la educación han sido elementos centrales de su formación como clase. Fragmentación porque tanto su comportamiento político como su aproximación más individualista a la

\footnotetext{
(*) Escuela de Sociología, Universidad Diego Portales. Santiago, Chile. Correo electrónico: Modesto.gayo@udp.cl

(**) Escuela de Sociología, Universidad Diego Portales. Santiago, Chile. Correo electrónico: Berta.teitelboim@udp.cl

${ }^{(* *)}$ Escuela de Sociología, Universidad Diego Portales. Santiago, Chile. Correo electrónico: marialuisa.mendez@udp.cl

${ }^{1}$ Este estudio se hizo en el marco del Proyecto $\mathrm{N}^{\circ} 1100523$ del Fondo Nacional de Desarrollo Científico y Tecnológico (FONDECYT), titulado: "La desigualdad cultural y sus determinantes. Un estudio sobre el gusto y la participación cultural en Chile". Además de reconocer este apoyo institucional, quisiéramos agradecer los comentarios de los evaluadores anónimos de este artículo, cuyas sugerencias contribuyeron a mejorarlo hasta su estado actual.
} 
actividad cultural estarían asociados a una importante heterogeneidad interna. Sin embargo, sobre todo a propósito de este segundo aspecto, su eventual división, existe mucha más información sobre su comportamiento político que sobre el que atiende a los gustos y la participación cultural. El presente artículo toma como punto de partida las contribuciones previas y estudia para el caso chileno los perfiles de exclusividad y fragmentación. En el caso del primer perfil, enfrentamos a la clase media con lo que aquí entendemos como la clase obrera, a partir de cuya comparación pueden identificarse orientaciones hacia la práctica cultural muy diferentes entre ambas clases sociales. En lo que respecta a la fragmentación, estamos ante una temática mucho menos explorada en la sociología y aquí proponemos una división que separa con claridad a los "profesionales" de las otras categorías ocupacionales que conforman esta clase. Además, estudiamos la estructura social de la fragmentación de la clase media.

\title{
Palabras clave:
}

Clase media, clase obrera, consumo cultural, capital cultural, alta cultura, cultura popular.

\begin{abstract}
The sociological literature about the (upper) middle class, developed during the second half of the Twentieth century, has allowed us to have a detailed knowledge about its political and cultural behavior, particularly for the cases of Western Europe and the United States. From this line of work, we can draw two important ideas which have contributed to shape the image of this social class: exclusivity and fragmentation. Exclusivity because their members have been prone to support political standings similar to those of the highest social classes in society, and refinement and education has been central components in its formation as a social class. Fragmentation because its political behavior and a more individualistic approach to cultural practice would be associated to a significant heterogeneity. However, above all regarding this latter characteristic, that is, its internal division, much more information is available about its political behavior than the one that has been produced looking at tastes and cultural participation. This article takes these contributions as a point of departure and it studies exclusivity and fragmented profiles in the case of Chile. As regards the former profile, exclusivity, the middle class is compared with the working class, and from this comparison different orientations towards cultural practice are identified. On the other hand, in relation to the "fragmentation" thesis, this is a topic about
\end{abstract}


which research is not so abundant, and here it is proposed a divide that makes a very clear separation between "professionals" and all the other middle class occupational categories. Besides that, the social structure of the fragmentation of the middle class is also studied.

Keywords:

Middle class, working class, cultural consumption, cultural capital, high culture, popular culture.

\section{LA CULTURA DE LA CLASE MEDIA}

\subsection{Notas introductorias}

Desde un punto de vista cultural, la emergencia de la clase media tendría consecuencias enormes en las sociedades occidentales, al menos desde finales del siglo XIX. Bien sea la misma entendida como una nueva clase acomodada, como podrían ser los casos de Inglaterra y Francia, bien se refiera a una nueva masa, antes existente pero de menor tamańo, que accede a un consumo crecientemente ocioso, sin duda su aparición acompañó una revolución del mundo artístico y de la cultura popular. Con respecto a lo primero, la producción de arte, las vanguardias pictóricas y la proliferación de literatura no podrían ser cabalmente comprendidas sin tomar en consideración el surgimiento y sobre todo expansión de un mercado que no sólo demandaba belleza en la vida cotidiana, sino que proveía a un considerable número de mentes creadoras de un medio de vida ajeno, o más independiente, de la demanda tradicional del Estado, las órdenes religiosas o los mecenas terratenientes o ligados a exitosas iniciativas empresariales ${ }^{2}$. En relación con lo segundo, la cultura popular, el recientemente elevado nivel económico y la igualmente creciente y considerable sofisticación cultural de una importante parte de la sociedad propia de mediados del siglo XX en adelante dio pábulo a una

\footnotetext{
${ }^{2}$ Este encaje entre oferta o producción, por un lado, y demanda, consumo o práctica, por el otro, es propuesto por Pierre Bourdieu en el libro Las reglas del arte (2005). Así se observa cuando se sostiene que "de igual modo que no cabe imaginar hoy el cine de ensayo sin un público de estudiantes y de intelectuales o de aspirantes a artistas, de igual modo que no se concibe la aparición y el desarrollo de un vanguardia artística y literaria en el transcurso del siglo XIX sin el público que le garantiza la bohemia literaria y artística concentrada en París"(2005: 373); aunque, en este punto preciso, argumenta que este público es "demasiado pobre para poder comprar" (2005: 373).
} 
imparable transformación y diversificación de la oferta cultural para jóvenes, que después serían adultos, los cuales encontraron en nuevas formas de ocio identidades personales que, en mayor o menor medida, contribuyeron a dar sentido a sus vidas. Así, las nuevas generaciones no hablarían solamente de la guerra, la religión o los problemas económicos, sino también, o quizás más, del rock-and-roll, los viajes y sus intereses estéticos. Con ello, obviamente, no queremos sostener que la clase media sea el factor que provocó todos estos cambios, pues hay otras razones que tienen que ver con transformaciones tecnológicas, nuevas propuestas filosóficas y novedades políticas, pero es sin lugar a dudas un acompañante esencial del cambio cultural en el siglo XX y de la sociedad en la que vivimos hoy día ${ }^{3}$.

Si bien parece estar claro que la aparición de la clase media tuvo una importante influencia en el desarrollo cultural, no existe un acuerdo igual de amplio, por más tratado que este tema haya sido, sobre la exclusividad o masividad de las prácticas de esta clase social. Así, habiendo sido habitual asociarla con la sofisticación y la legitimidad cultural, tesis cuyo representante por antonomasia es Bourdieu (1979), al menos en las últimas dos décadas, dicha propuesta ha sufrido fuertes embates liderados, de un modo general, por una sociología cada vez más desinteresada de los fenómenos de clase, y, de forma más específica, por las ideas de la globalización (Castells, 2003), la individualización (Beck y Beck-Gernsheim, 2002) y, finalmente, el omnivorismo cultural (Peterson y Kern, 1996), todos ellos presentados como tendencias características de las sociedades post-industriales finiseculares.

No obstante, se haya debilitado o no la relación entre posición socioeconómica y cultura, ha seguido siendo claro que los patrones culturales de la clase media han continuado representando uno o varios modelos normativos. En general, ello se debe a que se ha visto en el aprendizaje de cierta sofisticación una forma de capital cultural que otorga ventajas a aquéllos que la interiorizan y la realizan en sociedad (Bernstein 1989; Devine, 2004; Bourdieu, 1979, 1989). En definitiva, siendo las posiciones identificadas con la clase media las consideradas como más ventajosas en una sociedad, dejando de lado la minúscula aunque poderosa élite, se prestó atención a sus habilidades y obviamente al proceso histórico a través

\footnotetext{
${ }^{3}$ La literatura que relaciona la clase social y la cultura muestra con mucha contundencia como hay importantes desigualdades en la práctica cultural y, en particular, existiendo éstas, como los miembros de la clase media sobresalen por su mayor activismo cultural (Peterson y Kern, 1996; Bennett et al., 2009; Gayo et al., 2009; entre muchos otros).
} 
del cual las mismas son socialmente producidas. Esto significa que la cultura de la clase media en un momento dado es el resultado de un continuo variable de disputas individuales socio-institucionalmente organizadas, y sus representantes serían los ganadores en tales batallas. Pero este logro cultural no puede ser entendido simplemente como un resultado, sino que se trata al mismo tiempo de una herramienta cuyo dominio o acumulación otorgará a sus portadores ventajas sobre otros menos aventajados, esto es, la cultura estará en el centro de las luchas por y las relaciones de poder en la sociedad.

\subsection{El perfil de la cultura de la clase media}

Si efectivamente hemos sido capaces de hablar de la presencia de una cultura de clase media, parece que sería importante especificar cuál sería su perfilí. En este sentido, si atendemos a los resultados del estudio realizado por Bennett et al. (2009), podríamos caracterizarla como más participativa, parcialmente exclusiva, tolerante y reflexiva 5 . Para entender esta afirmación, debemos saber que el otro con el cual se compara es la clase obrera. Por tanto, en primer lugar, los individuos pertenecientes a esta clase tenderían a participar en un número mayor de actividades culturales. En segundo lugar, se muestran relativamente más propensos a optar por algunas prácticas que comparten en limitada medida con las personas que disfrutan de menor acomodo cultural y material en la sociedad. Sin embargo, esa diferencia no obstaculiza el hecho de que muchas de sus actividades culturales son similares a las practicadas comúnmente por estos últimos ${ }^{6}$. En tercer lugar, sería un grupo tolerante porque manteniendo cierta exclusividad, o lo que podríamos entender como sofisticación, comparte algunas prácticas, como queda dicho, con la clase obrera y se muestra reacio a censurar la cultura popular. En los últimos años esta tolerancia ha sido conceptualizada como "omnivorismo cultural". Finalmente, se constataría un grado

\footnotetext{
${ }^{4}$ Es evidente que hablar de "cultura" no es muy específico y frecuentemente es un concepto demasiado abstracto para ser operativo o entender procesos particulares. Nosotros no proponemos resolver este problema a través de la postulación y defensa de una definición de "cultura", sino de la observación y estudio de un fenómeno concreto como es el de los patrones de gusto y participación culturales. En este sentido, compartimos la conclusión de Adam Kuper (2001), aplicable a la sociología, cuando se sostiene que " cuanto más se considera el mejor trabajo moderno de los antropólogos en torno a la cultura, más aconsejable parece evitar semejante término hiperreferencial y hablar con mayor precisión de conocimiento, creencia, arte, tecnología, tradición, o incluso ideología"(12).

${ }^{5}$ Un estudio en el que ya se adelantan ideas similares es el de Roberts (2004). "The middle classes do more of most things. The big exception is television viewing... Nowadays the middle classes are leisure omnivores... They are no longer exclusively highbrow" (65). Algunos ańos antes, distinguiendo entre los patrones de ocio de la clase media y la clase obrera, el mismo autor afirmó: "Whilst the working classes tend to spend their leisure passively, being entertained by professional sportsman and broadcasters or just relaxing, the middle classes are more inclined to adopt active leisure interests" (1970: 27).

${ }^{6}$ Para el caso chileno, sobre este punto, véase Gayo (2011).
} 
alto de reflexividad a través de la manifestación expresa de conciencia sobre las categorías sociales empleadas para entender las diferencias y gustos culturales.

Hasta ahora hemos hablado de la cultura de la clase media, como si estuviéramos frente a una unidad, y quizás con la tentación de ver en ello algo estático. Con respecto a cualquiera de estas dos afirmaciones debemos tener cautela. En primer lugar, porque la cultura, y en particular las prácticas culturales, han mostrado fuertes cambios a lo largo del tiempo, tanto en su realización como en su valoración, lo cual no parece que haya estado siempre vinculado al binomio distinción/alta cultura $^{7}$. En segundo lugar, porque después del énfasis en el crecimiento de la clase media propio de los ańos 50 y 60, la literatura sociológica, sobre todo desde los años 80 , expresa un cuasi consenso a favor de la heterogeneidad de la misma ${ }^{8}$. En este sentido, se entiende que los tres principales grupos que la componen son los profesionales, los directivos y los trabajadores de cuello blanco o lo que podríamos llamar empleados no manuales, administrativos o de oficina. El sentido común en países como Chile nos llevaría a pensar que la clase media se refiere de forma preferente a los últimos, pero, sin embargo, la reflexión sociológica, de forma masiva en las últimas tres décadas, se ha enfocado en los dos primeros grupos, los que se aceptan como los grandes vencedores de la post-industrialización (Bell, 1974). Si existe, por tanto, consenso sobre quienes forman parte de esta clase, estaría compuesta sin lugar a dudas por los primeros, existiendo serias dudas o falta de acuerdo sobre la pertenencia a la misma del empleo de "cuello blanco".

Para el caso chileno, estudios recientes sobre participación cultural y gusto muestran que un entendimiento cabal de este fenómeno debe subrayar la influencia en su estructuración de las desigualdades económica y cultural, junto a la edad (Gayo, Teitelboim y Méndez, 2009; Gayo y Teitelboim, 2009a; Gayo y Teitelboim, 2009 b) ${ }^{9}$. Si adoptamos el concepto más restrictivo de clase media, el cual incluiría a directivos y profesionales, en Chile estaríamos ante el grupo de personas que tiene mayor propensión a participar en actividades culturales y manifiesta una

\footnotetext{
${ }^{7}$ Un estudio que plantea dudas sobre la necesaria centralidad del capital cultural es el de Bennett et al. (1999) sobre Australia, en el cual concluyen que "In general, we hypothesise; it is economic capital and social capital that play the major role in the generation and reproduction of class inequality in Australia" (268). Asimismo, Michelle Lamont (1992) en un estudio comparado entre Estados Unidos y Francia, sostiene que los patrones valorativos de las clases medias altas de estos dos países son diferentes, siendo la francesa la que presta mayor atención al capital cultural como elemento de distinción.

${ }^{8}$ Un análisis detallado de la tesis de la heterogeneidad puede ser encontrado en Méndez y Gayo (2007: 134-138).

${ }^{9}$ Sin ser muy abundante, hay más literatura para el caso chileno y una primer revisión de la misma puede ser consultada en Gayo et al. (2009).
} 
tendencia notablemente mayor a poseer bienes de esta naturaleza. En definitiva, son los que pueden ser asociados con la cultura comúnmente considerada más elevada: van más a la ópera, al teatro, al cine, compran más libros, tienen más pinturas y esculturas, viajan con mayor frecuencia tanto dentro como fuera del país, entre otras cosas. Por tanto, si algo caracteriza a esta clase media, al menos en términos relativos, eso es su activismo cultural.

Con estas reflexiones en mente, en este artículo afrontamos las siguientes tareas. En primer lugar, ofrecemos información sobre el método y los datos relacionados con la operacionalización que está en la base de esta investigación. En segundo lugar, especificamos un poco más las características de la diferencia entre la clase media y aquellas ocupaciones que se encontrarían por debajo de la misma, lo que históricamente vino siendo conocido como "clase obrera". Está claro que ésta es sólo una parte del todo, pero la reflexión sobre la misma nos servirá para hablar con suficiente profundidad y extensión de la desigualdad, sin necesidad de referirnos a todos los que sufren desventajas. En tercer lugar, prestamos atención a la posición de las personas identificadas con la clase media, de modo de plantear dudas sobre su supuesta posición "en medio". En cuarto lugar, estudiamos las diferencias dentro de esta clase, atendiendo de esta manera a una de las grandes tesis sobre su naturaleza, según muchos fragmentada. Finalmente, en quinto lugar, nos centramos brevemente y de forma parcial en el debate sobre el consumidor cultural "omnívoro", y para ello presentamos los resultados de un análisis de datos en el cual se muestra las peculiaridades de la clase objeto de estudio.

\section{BREVE NOTA METODOLÓGICA}

Desde un comienzo, nuestro objetivo fue afrontar las características de participación cultural propias de las personas que habían mostrado una mayor propensión hacia el activismo de esta naturaleza. Un cuerpo de reflexión sociológica que encaja con este objetivo es el que se refiere a la clase media. Si bien no hay un acuerdo completo sobre la definición de esta clase social, ha sido habitual el uso de categorías ocupacionales para su operacionalización ${ }^{10}$. Sin embargo, para mostrar un alto grado de inducción y de ajuste al caso chileno, no adherimos a ninguna

\footnotetext{
${ }^{10}$ Las muy conocidas clasificaciones de clases de Erikson y Goldthorpe (1993) y E.O. Wright (1994) trabajan con características de ocupaciones.
} 
clasificación de clases sociales, y es por ello que adoptamos como punto de partida una tipología de ocupaciones lo más desagregada posible. Desde un punto de vista operativo, hicimos uso de la información que nos ofrece la Encuesta Nacional de Consumo Cultural del CNCA del año 2009, la cual venía codificada de acuerdo a la Clasificación Internacional Uniforme de Ocupaciones (CIUO) de la OIT de 1988. Tomamos a los ocupados, pues eran los únicos sobre los que teníamos la información necesaria para vincularnos con las aproximaciones desde las clases sociales. Esta fue nuestra población real de trabajo, y la razón por la cual nuestro $100 \%$ (véase tablas 2 y 3 ) debe ser entendido dentro de este procedimiento.

Después de un importante análisis descriptivo preliminar, el cual nos permitió tomar decisiones sobre la necesidad de agregar categorías ocupacionales, muy desagregadas en la versión original (ver tabla 1 en el anexo 1), hicimos un primer estudio mediante el empleo de la técnica estadística conocida como análisis de correspondencias múltiples. Dicho de forma muy breve, esta técnica permite estudiar las relaciones entre un conjunto variable, pero eventualmente amplio, de categorías de variables categóricas. De este modo, podemos obtener dos tipos de información. Por un lado, podemos saber la proximidad relativa entre las categorías, de tal modo que es posible, cuando comparamos dos de ellas (por ejemplo, los que van y no van a la ópera) extraer conclusiones sobre su probabilidad relativa de hacer una tercera actividad (por ejemplo, ver programas deportivos o no). Por otro lado, al igual que hacemos con el análisis factorial, nos permite estudiar las variables latentes o no observadas que explican (en principio, estadísticamente) las relaciones que estamos estudiando. Estas variables a menudo son conocidas como ejes, dimensiones o factores. De este estudio, se derivó el siguiente patrón, que mostramos en el gráfico 1. Lo que encontramos es principalmente dos grandes grupos de ocupaciones, las cuales encajan bien con definiciones o descripciones habituales tanto de la clase media como de la clase obrera. Si bien nosotros estábamos interesados en la clase media, era importante atender a su diferenciación con respecto a otras clases sociales, pues de otro modo no sería posible mantener su distinción, la cual es siempre relativa a lo que sucede en el conjunto de la sociedad. En la tabla 2 (ver anexo 1) se encuentra la información sobre la distribución de los grupos ocupacionales a los cuales se llegó después del estudio descriptivo original y la decisión sobre su inclusión en una clase particular, la cual fue adoptada tras el análisis de correspondencias que se hizo en primer lugar, tal y como hemos descrito. 
Los datos utilizados son los de la Encuesta Nacional de Participación y Consumo Cultural realizada en el año $2009^{11}$. Se trata de un estudio que tiene una muestra de 4.176 casos, el cual se realizó entre enero y abril de 2009 a la población de Chile mayor de 15 años. Para ello, con el objetivo de tomar en cuenta los diferentes territorios del país, se utilizó un método de estratificación geográfica, lo que atendió de forma prioritaria a la consideración de la variable regional. En cuanto a su contenido temático, los principales ejes de esta encuesta fueron los siguientes: 1. la participación en eventos artísticos y culturales (asistencia, frecuencia, lugar y medios de información), 2. el consumo de cultura envasada (libros, películas y música), 3. la infraestructura y equipamiento cultural, y 4 . los medios de comunicación de masas.

Gráfico 1. Agrupación de categorías ocupacionales de acuerdo con su práctica cultural

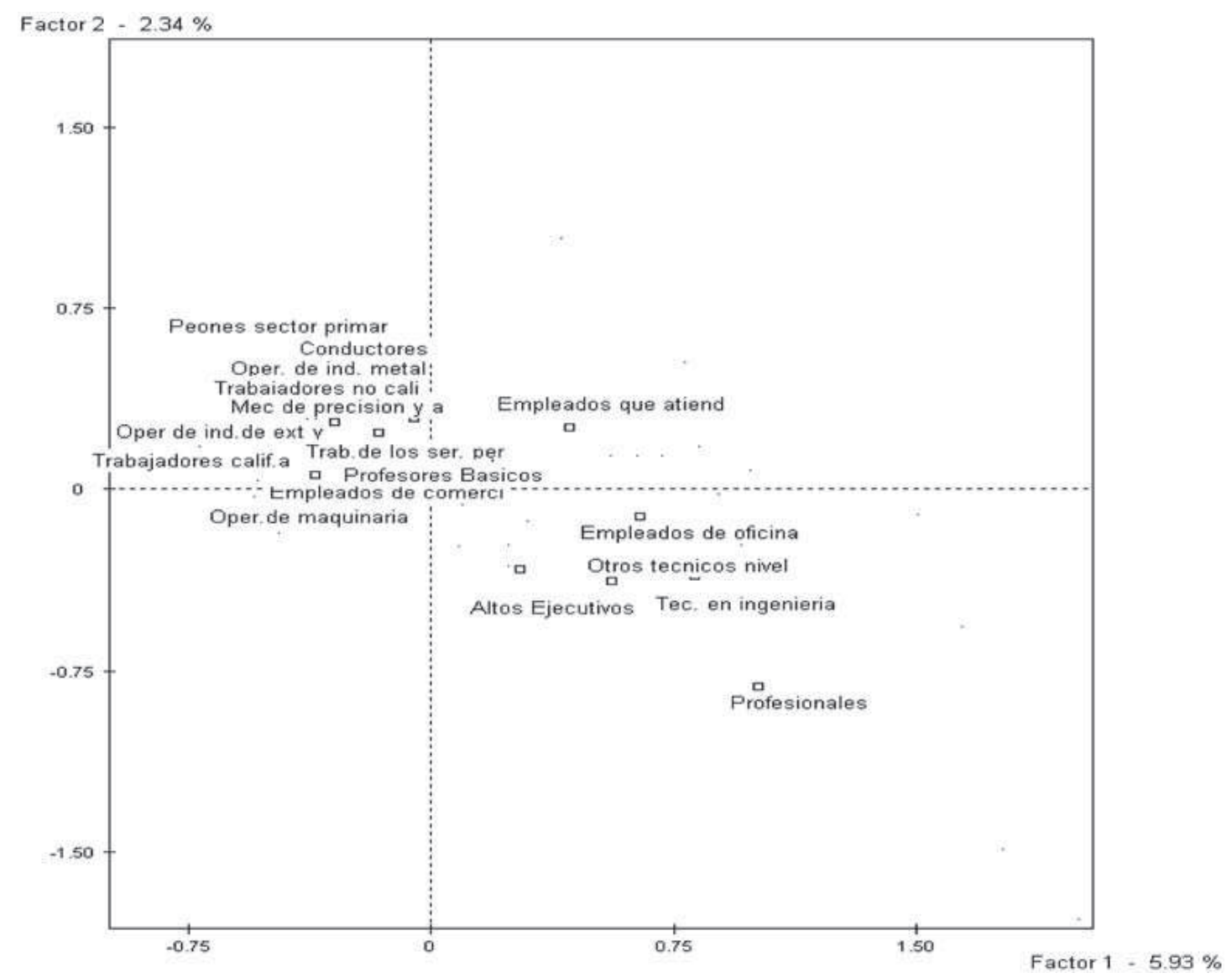

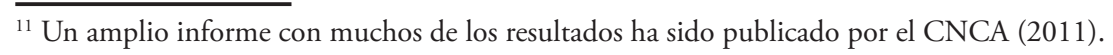




\section{LA DIFERENCIACIÓN SOCIAL}

Si la clase media tiene un otro, más que la clase alta o la burguesía, ése es la clase obrera. Históricamente, la primera ha venido siendo lo que la segunda no es. La diferenciación entre ambas ha dependido tanto de sus recursos económicos como de sus respectivos estilos de vida. Ser clase media ha sido muy frecuentemente una forma de ser y presentarse en sociedad (de ahí, por ejemplo, el término "whitecollar" frente a "blue-collar").

En esta línea, de acuerdo a Bourdieu (1979) habría coherencia u "homología" entre los perfiles culturales y las posiciones de clase de las personas. Eso significa que un trabajador con poco capital económico y educativo mostraría limitada propensión hacia el activismo cultural, sea éste la asistencia a eventos de esta naturaleza, el interés por adquirir nuevos conocimientos, o la compra de objetos con valor estético. Por el contrario, un empleado con elevado capital económico y cultural propendería, siempre en términos relativos, a desarrollar gustos exclusivos y sofisticados, a interesarse por desarrollar sus conocimientos y a incorporar en su vida una valoración positiva y un intento de realización efectiva de la belleza. En otros términos, el gusto estaría estructurado socio-económicamente. En lugar de pies de barro, los tendría de plomo ${ }^{12}$.

Si atendemos a este punto, en el caso chileno vemos (ver gráfico 2), mediante el uso del análisis de correspondencias múltiples ${ }^{13}$, que efectivamente existe una notable diferencia entre los patrones culturales de las ocupaciones más obreras y las que podemos asociar a la clase media ${ }^{14}$. Por tanto, la estructura de clase está fuertemente asociada a las prácticas culturales de los chilenos. Esto es muy importante porque se hace evidente que esta sociedad no puede ser solamente

\footnotetext{
${ }^{12}$ En este punto es muy importante subrayar que estamos hablando de diferencias en términos de prácticas y gustos, y no de la mayor o menor asignación de tiempo de las personas a las actividades de ocio, lo que sería notoriamente diferente. A modo de ejemplo, y con ánimo de seguir pensando la relación entre cultura y estructura, si nos referimos a esto último, Ken Roberts destacaba algunas décadas atrás el igualitarismo de la sociedad británica. Éstas son algunas de sus palabras al respecto: "The amount of leisure time that an individual enjoys does not depend mainly upon his occupational status. The extent of an individual's non-work social obligations such as family commitments makes more difference to the amount of free time that he has at his disposal... In this sense Britain can be described as a leisure democracy (1970: 10).

${ }^{13}$ Además de lo apuntado en la sección previa, para una breve explicación de esta técnica, nos remitimos a Gayo et al. (2009: 51-53).

${ }^{14}$ El gráfico muestra una importante diferencia entre ambas clases sociales. El solapamiento que se puede observar tiene relación con el hecho de que la mayoría de la población tiene bajos niveles de participación cultural, lo que iguala a parte de la clase media con los miembros de la clase obrera. Como pudimos observar en el gráfico 1, si atendemos a lo que sucede a los diferentes grupos ocupacionales, su posicionamiento en el espacio social es coherente con lo que aquí sostenemos.
} 
interpretada desde el éxito o el crecimiento económico, sino que la desigualdad afecta fuertemente a la cotidianeidad de las personas que en ella viven, y esto es así tanto para los ingresos, tema probablemente más recurrente, como desde el punto de vista de las disposiciones y comportamientos de sus miembros.

Gráfico 2. Posicionamiento en el espacio social de los miembros de las clases sociales media y obrera de acuerdo con su participación y gusto culturales

Factor $2 \cdot 233 \times$

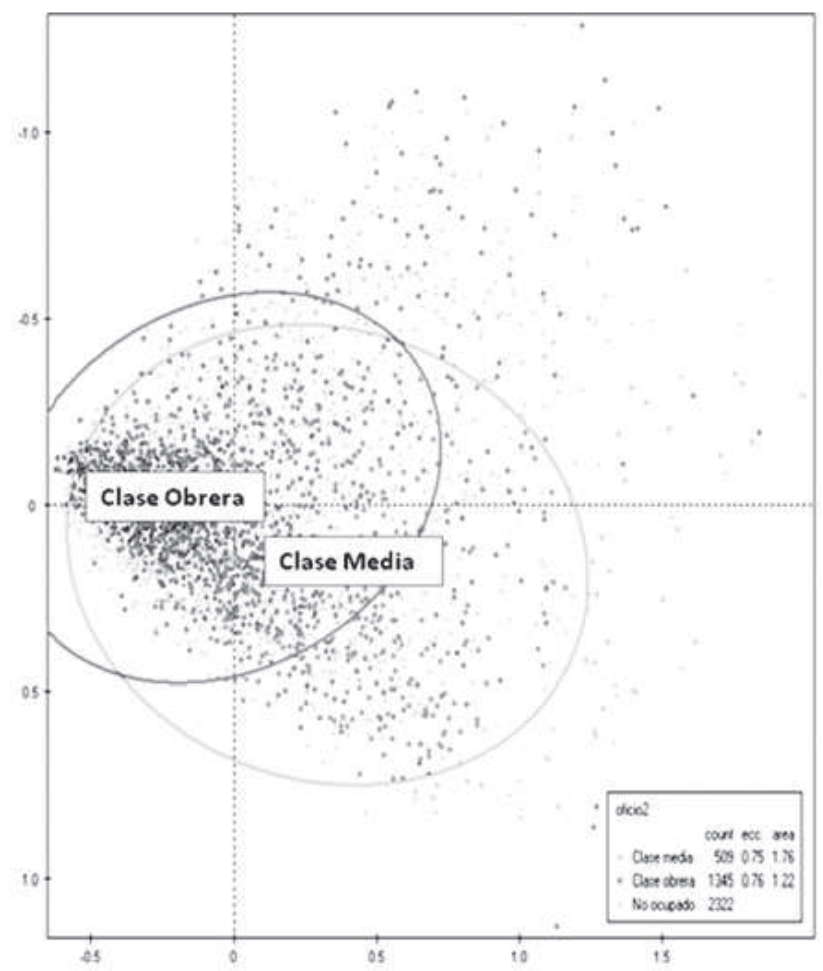

Tomando en cuenta la diferencia entre las clases aludidas, debemos preguntarnos “¿cuáles son las características de esta diferenciación?”. Hay varias muy significativas, las que presentamos a continuación. En primer lugar, la clase media es más participativa, y esto en dos sentidos. Por un lado, participa en más actividades culturales, y, por otro, su rango desde un punto de vista cualitativo es mayor. Es decir, hacen más cosas y con mayor frecuencia. En segundo lugar, tal y como se puede observar en el gráfico 2, mantienen una zona de exclusividad en la que no entra la clase obrera. En otros términos, lo que hacen los miembros de esta última clase lo hacen todos, mientras que hay patrones que son propios de la clase media. Aquí hay objetivamente un área de distinción, por utilizar 
el célebre concepto bourdieuano, si bien esto en sí mismo no nos habla de la subjetividad de los que realizan dichas prácticas. En tercer lugar, de un modo más específico, las personas de clase media destacan, entre otras cosas, por las siguientes actividades y preferencias: asistir a exposiciones de pintura y fotografía, ir al teatro y a espectáculos de ballet y danza, el gusto por las músicas clásica o la ópera, la folclórica, el rock y las baladas románticas, las actuaciones de malabarismo y títeres, la asistencia frecuente al cine, y dentro de los tipos del mismo, prefieren los dramas, el cine arte y las películas históricas, leen libros regularmente, siendo sus estilos preferidos las novelas, los libros de autoayuda y los de tecnología y ciencia, van a museos de arte, históricos y de ciencia, ven televisión con frecuencia, pero menos que la clase obrera, poseen artesanías, pinturas originales, esculturas, grabados y libros de colección, son selectivos leyendo los diarios, prefiriendo las secciones de opinión y de cartas al director, junto a las de noticias internacionales, política nacional y la dedicada a la economía y el mundo laboral, escuchan música en la radio, manifiestan una mayor preferencia relativa por las revistas de diseño y decoración, actualidad política, cultura y artes, y las científicas y tecnológicas, utilizan internet para comunicarse y buscar información, destacan por el número de libros que poseen, viajan dentro y fuera de Chile, visitan sitios patrimoniales, ferias de artesanía, asisten a conferencias, van a parques de diversiones y zoológicos, y realizan actividades deportivas.

En cuarto lugar, en lo que se refiere a la clase obrera, podemos observar como estos individuos muestran, siempre en términos relativos, el siguiente patrón de participación y gusto: no van a exposiciones ni a obras de teatro, no asisten a espectáculos de danza y ballet, les gusta la música de los tipos sound, salsa o cumbia, no suelen ir a conciertos, ni el malabarismo ni los títeres los atrae especialmente, van relativamente poco al cine, y con respecto al mismo tienen preferencia por el de acción, las películas cómicas o de humor, y las de suspenso, ven menos videos que los miembros de la clase media, no tienden a leer libros, y si lo hacen, entre sus preferidos están los cuentos y los religiosos, no visitan museos, son los que ven más televisión, poseen pocos objetos culturales (pinturas, grabados...), leen menos los diarios y cuando lo hacen, se orientan a la lectura de las secciones de crónica nacional policial y deportiva, destacan en el uso de la radio para escuchar programas deportivos, no leen revistas, no hacen uso de internet, no tienen libros o los acumulan en escaso número, no viajan ni fuera ni dentro del país, y van al estadio. 


\section{UNA CLASE QUE NI ES LA MAYORÍA NI ESTÁ EN EL MEDIO}

Generalmente, durante la segunda parte del siglo XX, la presencia de la clase media ha sido asociada con mayores oportunidades de estabilidad política, y en particular ha sido entendida como una condición para el desarrollo de democracias duraderas. Por tanto, la clase media ha formado parte de la normatividad y normalidad democrática (Lipset, 1987; Chauvel, 2006). Este entendimiento supone que esta clase ha sido considerada un punto de equilibrio social, entre modelos de alta concentración de riqueza, elevadas inequidades y fuerte polarización política, por un lado, y otros notablemente igualitarios y con limitado crecimiento desde un punto de vista económico, al mismo tiempo que monolíticos políticamente. Sin embargo, desde una perspectiva cultural difícilmente podemos sostener simplemente que la clase media sea un lugar intermedio, o de paso (Bourdieu, 1979). Salvo que insistamos en subrayar la presencia de una élite, siempre minúscula, la cultura de la clase media es tanto un modelo normativo, como un patrón, o conjunto de patrones, con un importante tinte de exclusividad, que configura en su totalidad el polo culturalmente supra-ordinado de la sociedad chilena. Frente a ella, en desventaja o subordinación, observamos a una clase obrera menos propensa a la participación y de gustos masivos. ¿Qué está implicado en estas tesis? Derivado de la identificación de estas dos grandes clases, producto de la agregación de ocupaciones ${ }^{15}$ (ver tablas 1 y 2 en anexo 1 ), podemos sugerir la presencia de un escenario de polarización que nos habilita para entender conflictos sociales que pudieran tener su base en distinciones culturales y no sólo económicas, aun sabiendo que ambas dimensiones están fuertemente relacionadas en Chile, tal y como demuestran estudios previos (Gayo et al., 2009). A estas alturas, a través de otras investigaciones a nivel comparado, ya sabemos que las desigualdades económicas no siempre están asociadas a las mismas distinciones culturales (Lamont, 1992; Gayo et al., 2011), y el estudio de estas últimas es lo que nos interesa aquí. El fenómeno de la estructuración de las prácticas culturales entorno a dos grandes clases sociales es muy importante. Esto es debido a que ha llegado a entenderse como evidente, quizás incluso peligrosamente, que las

\footnotetext{
${ }^{15}$ Ken Roberts (1970) defendió, en un texto ya antiguo, que era inadecuado entender las prácticas de ocio como un producto de la estructura ocupacional. En su lugar, sugirió la importancia de los patrones de ocio en la forma de entender su trabajo. Así, afirmaba que "it can be argued that for many people leisure has now become such a central and dominant part of their lives that is their behavior and attitudes towards work that are determined by their leisure rather than the other way round" (1970: 25).
} 
nuestras son sociedades de clase media, y esto ha sido aceptado no solamente por gran parte de la población de diferentes sociedades, sino igualmente por multitud de académicos (Mills, 1959; Lipset, 1987; Savage et al., 1995). Esta evidencia contiene un peligro: el de considerar a la clase media, o a las ocupaciones asociadas a la misma, como una posición neutral en el espacio social. Una aproximación ingenua, con este contenido último, podría sostener que la élite es la responsable de las desigualdades culturales y la clase media es el lugar al que todas las personas miembros de una sociedad pueden y deberían llegar. La clase media, así, es un estado ideal de una comunidad, a cuya presencia se asociarían valores como la integración y la igualdad. En contra de esta idea, nuestra investigación no sólo ha mostrado consistentemente, en este y otros trabajos, que un entendimiento de la participación y el gusto culturales en Chile debe incorporar a la clase social como variable clave, sino que la clase media muestra un patrón más participativo y exclusivo que la clase obrera, lo que sin duda tenderá a hacerse evidente en la vida cotidiana. Además, debemos enfatizar que la clase media, o la extensión del patrón cultural asociado con la misma, no incluyen a la gran mayoría de la población ocupada, sino que incorpora entre un 25 y un 30\% de la población (ver tabla 3). En este caso, se trata únicamente de los ocupados, pero cifras similares pueden ser encontradas en otros estudios (Gayo y Teitelboim, 2009a). Siendo este el cálculo más optimista, debemos entender que los perfiles más exclusivos concentran a porcentajes de población considerablemente menores. Eso significa no sólo que la clase media es representante de gustos y prácticas minoritarias y socialmente selectas, sino que en su interior podemos encontrar una importante fragmentación, y eso es lo que estudiamos en el próximo apartado.

Tabla 3. Porcentajes de las clases media y obrera

\begin{tabular}{|l|c|c|}
\hline & Clase media & Clase obrera \\
\hline Población ocupada & $27.8 \%$ & $72,2 \%$ \\
\hline
\end{tabular}

(Elaboración propia a partir de encuesta nacional de consumo cultural del CNCA, 2009).

\section{LA FRAGMENTACIÓN INTERNA: SIMILITUDES Y LUCHAS}

La idea de que la clase media incluye un conjunto heterogéneo de personas y que por ello mismo está fragmentada ha recorrido la literatura sociológica al menos desde los ańos 50 del siglo pasado, pudiendo ser un primer ejemplo de ello la 
obra de Wright Mills White Collar (1959) ${ }^{16}$. Si bien es cierto que en dicho estudio tal heterogeneidad no fue claramente retratada, se presenta a esta clase como un cajón de sastre del empleo no manual en el que la diversidad (en jerarquía, ingresos, funciones...) era más la norma que la excepción. El estudio de esta clase en décadas posteriores ha venido a reforzar esta idea de división, y hoy, aun con alguna resistencia relativa en obras importantes (Erikson y Goldthorpe, 1993), es un lugar común en la academia hablar sobre las diferencias existentes entre sus componentes ${ }^{17}$.

La diversidad ha estado referida muy comúnmente al comportamiento político, bien sea éste entendido en términos de voto (Heath y Savage, 1994; Brooks y Manza, 1997), bien estemos hablando de movimientos sociales (Kriesi, 1989; Rootes, 1995), y también ha alcanzado al terreno cultural. Un ejemplo insigne de esto es la obra de Pierre Bourdieu. En la misma, específicamente en La distinction (1979), se defendía que la clase dominante, lo que aquí podríamos entender como clase media en la acepción que propusimos, está escindida o dividida. En concreto, tendría una estructura en cisma configurada a partir de la composición de los capitales, cultural y económica, de las posiciones que la componen. Si bien en su conjunto éstas constituirían un continuo, acorde a la lógica de distribución de los capitales, en líneas generales, podríamos reducir su complejidad a dos grandes grupos. Por un lado, tendríamos a los que destacan por su acumulación de capital económico, como los industriales y los profesionales liberales, mientras, por otro, se encontrarían los que concentran de forma más importante, y por tanto dependen de, el capital cultural, entre cuyos representantes estarían los profesores de universidad y enseñanza secundaria junto a los artistas.

En buena parte de la investigación y la literatura que ha estudiado la heterogeneidad dentro de la clase media, entre los que están los casos comentados más arriba, se puede observar la gran importancia que se ha atribuido a la ocupación como un factor que contribuye a dividir a esta clase social. Esto es coherente con el desarrollo del campo de investigación sobre la clase media en Chile, donde hay una tradición

\footnotetext{
${ }^{16}$ Otro ejemplo de una obra de la misma época que enfatiza la heterogeneidad, en este caso política, es la de Bonham (1954). En la misma el autor distingue entre dos grupos principales. Por un lado, estarían los directivos y profesionales, más conservadores, y, por otro, encontraríamos a los trabajadores de cuello blanco, más propensos a apoyar a la izquierda política y, en definitiva, más próximos a la clase trabajadora.

${ }^{17}$ Ken Roberts (1970) ya advierte que ni la clase media ni la obrera deben ser consideradas entidades sociales homogéneas.
} 
de estudios, provenientes tanto de la sociología como de la historia, que se ha centrado, por un lado, en su carácter urbano y su crecimiento en función del empleo público (Filgueira y Geneletti, 1981; Martínez y Tironi, 1985; León y Martínez, 2001; Salazar y Pinto, 1999-2002; Barozet y Espinoza, 2008; Candina, 2009), y por otro, de forma complementaria, en la medición de la clase media a partir de los ingresos y la ocupación (León y Martínez, 2001; Torche y Wormald, 2004; Torche, 2005; Franco et al., 2007). A este respecto, en el caso chileno, los estudios que adoptan la perspectiva cultural han sido enfáticos en mostrar "la fuerte dispersión y heterogeneidad de experiencias sociales observables en el seno de un mismo grupo social" (Araujo y Martucelli, 2011) como es la clase media. Estos estudios ponen énfasis en el conjunto de contenidos simbólicos asociados a la clase media, en cuanto identidad, categoría o posición en la estructura social. Por una parte, es posible identificar investigaciones de corte fenomenológico que atienden a los significados asociados a la movilidad social (Méndez, 2003), estudios de orden más pragmático que exploran la producción de categorías residenciales en la clase media (Ariztía, 2007), o trabajos que desarrollan la perspectiva de los repertorios culturales para comprender la construcción de esta identidad (Méndez y Barozet, 2012) ${ }^{18}$.

En coherencia con lo anterior, nosotros comenzamos por atender a las diferencias que pudieran ser encontradas entre los grupos ocupacionales que incluimos dentro de esta clase. Para ello, como hicimos previamente, recurrimos al análisis de correspondencias múltiples, pero en lugar de seguir utilizando todos los casos, decidimos seleccionar únicamente a los encuestados de clase media, de tal modo que pudiéramos estudiar la configuración de un espacio social constituido en base exclusivamente a sus preferencias. Esta forma de operar nos brindó la oportunidad de analizar en detalle los agrupamientos de gustos y prácticas culturales de los individuos de esta clase, evitando las dificultades de visualización que plantearía haber utilizado todos los casos, dado que esto hubiera restringido el espacio para la clase media a una parte del gráfico y no a su conjunto ${ }^{19}$. Los datos muestran que si bien no es posible desechar la ocupación como una fuente importante de división dentro de la clase media, su capacidad explicativa, tal y como apunta Roberts

\footnotetext{
${ }^{18}$ En el caso de la investigación del caso chileno, Méndez encuentra similitudes que apuntan a enfatizar tensiones entre identidades de clase media auténticas y menos artificiales, como por ejemplo tensiones entre nociones de autenticidad versus intelectualismo entre sectores medios "gentrificados" (Santiago antiguo), autenticidad versus consumismo, o autenticidad versus arribismo (Méndez, 2007; Méndez, 2008).

${ }^{19}$ Para ver el gráfico del conjunto del espacio social chileno de acuerdo a las prácticas y gustos culturales, véase Gayo et al. (2009).
} 
(1970), es limitada y es importante incorporar variables no siempre consideradas como son el género y la edad. En efecto, nuestro estudio muestra que, al igual que otros desarrollados recientemente (Sémbler, 2006; Barozet y Espinoza, 2008; Franco et al., 2011), es preciso introducir perspectivas multidimensionales para hablar de clase media/clases medias en Chile, y en ese sentido, la heterogeneidad cultural dentro de la clase media tiene directa relación con estos dos últimos factores, siendo el género una categoría particularmente clave para comprender su fragmentación. Es por ello que es fundamental entender que las fracturas dentro de las clases no siempre tendrán que ver con divisiones ocupacionales en su interior, como ha sido habitual pensar en la sociología de corte o inspiración estructuralista y con, explicitada o no, preocupación por el marco impuesto por las sociedades industriales. Un paso más allá sería pensar que las clases sociales, en lo que afecta a la cultura, no tienen que ver necesariamente con las ocupaciones, sino con otras variables, lo cual es plausible pero aquí no abordado. En cualquier caso, ello se agrega al hecho de que todo parece indicar que, al igual que sucede para el conjunto del espacio social (Gayo et al., 2009), la gran variable explicativa es el nivel educativo.

Entonces, ¿qué características específicas tiene la fragmentación de la clase media que hemos anunciado? Para responder apropiadamente a esta pregunta, dividiremos nuestra respuesta en dos partes. En la primera, mostraremos algunos agrupamientos de categorías en el espacio a partir de los cuales parecen emerger algunos patrones bien diferenciados relativos a estilos de vida. En la segunda parte, propondremos algunas fracturas asociadas a las variables estructurantes mencionadas en el párrafo previo, y que deberían contribuir a una explicación cabal de los patrones observados previamente. Por lo que respecta al modo en que los gustos y actividades se agrupan (véase gráfico 3), podemos hablar de cinco grupos ${ }^{20}$. El primero (cuadrante superior izquierdo) refleja el interés por actividades culturales populares: los tipos de música sound, salsa o cumbia, bajar música a través de internet y la ostensiblemente reducida participación en un amplio conjunto de otras actividades. El segundo agrupamiento (cuadrante inferior izquierdo) parece tener en su centro el inactivismo cultural como eje de

\footnotetext{
${ }^{20}$ La lectura del gráfico producto del análisis de correspondencias múltiples es abierta, flexible y pautada. Abierta porque el gráfico suele admitir varias interpretaciones. Flexible porque éstas, cualquiera sean, están, casi por definición, llenas de áreas grises cuya comprensión es susceptible de re-interpretaciones. Por último, es pautada porque la apertura y la plasticidad de la interpretación no implica en ningún caso que cualquier cosa pueda ser dicha. La importancia de los patrones que encontramos tienen directa relación con nuestra aproximación intelectual, y son en este sentido relativos a la misma, pero la presencia de aquéllos es producida independientemente del interés del analista.
} 
su comportamiento. Un tercer grupo de personas serían especialmente propensas al seguimiento de la información deportiva tanto en radio como en los diarios, asistirían al estadio (de fútbol), irían al zoológico, a los parques de diversiones y les gustarían los estilos de música reggaetón o hip-hop. En cuarto lugar, aparece un conglomerado de actividades y gustos el conjunto de los cuales constituye con claridad lo que habitualmente se entiende por "alta cultura". Se trata de personas que viajan dentro y fuera del país, visitan sitios patrimoniales, asisten a conferencias, ven cine arte, tienen libros de colección, pinturas originales, grabados y esculturas, poseen un gran número de libros, visitan museos de ciencias, van con frecuencia al teatro y al cine, y manifiestan una propensión relativamente alta a apreciar positivamente tanto el ballet y la danza como la música clásica y la ópera. Finalmente, un quinto agrupamiento de actividades, vincularía el seguimiento de las revistas de actualidad política con las de diseño y decoración, a lo que se sumaría el gusto por las secciones de diario dedicadas a las noticias internacionales y las películas de cine de género dramático. A ello también parecen estar asociados el aprecio por la pintura y los museos de arte.

Gráfico 3. Patrones de participación y gusto de la clase media

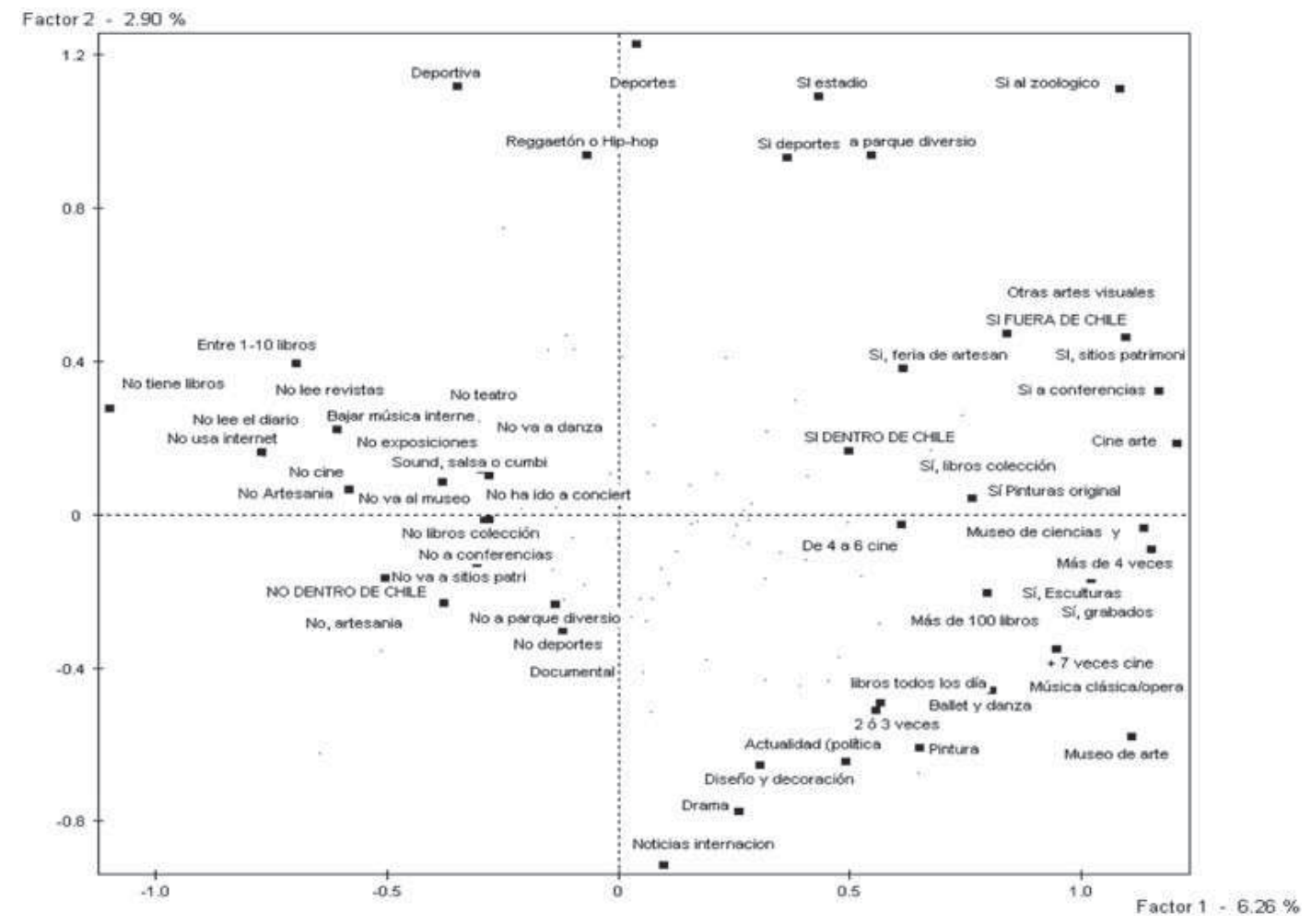


¿Cuáles son las variables que hemos encontrado que pueden tener relación con los patrones de práctica cultural presentados? Si atendemos al gráfico $4^{21}$, tendríamos que hablar de las siguientes. Primero, con respecto al eje 1, destacan el grupo socio-económico y el nivel educativo, tal y como muestran estudios previos (Gayo et al., 2009). Esto significa que dentro de la clase media también correlacionan positivamente los dos factores mencionados y son la principal fuente de estructuración de los patrones culturales, así como lo son para el conjunto del espacio social. A este respecto, desde el punto de vista de la reflexión sobre la heterogeneidad de esta clase entendida desde las ocupaciones, lo que ha sido la línea de aproximación más habitual, podemos constatar que no estaríamos meramente frente a una diferencia entre profesionales y ejecutivos o cuadros, sino ante una división que enfrentaría a los primeros con los segundos junto a todas las otras ocupaciones, entre las cuales estarían varios tipos de técnicos y los empleados de oficina. Este vínculo tan fuerte entre nivel educativo, grupo socio-económico y ocupación pareciera sugerir que más que ante una divisoria ocupacional, estaríamos ante una fragmentación con base en el nivel educativo, variable de acuerdo con la cual los profesionales muestran los grados más elevados entre todas las ocupaciones. Sin desechar totalmente que pueda haber subculturas profesionales u ocupacionales, la heterogeneidad cultural de la clase media se fundamenta más en las desigualdades educativas que en ningún otro factor. En cualquier caso, aunque pueda resultar evidente, conviene decir que los más educados y con mayores recursos económicos son los que presentan un perfil más participativo y más vinculado a la "alta cultura". Un segundo factor es la edad, con respecto a la cual debemos constatar su comportamiento claramente diferente al que presentaba para el conjunto del espacio social. Ahora sucede exactamente lo contrario de lo que habíamos sostenido en estudios previos (Gayo et al., 2009), es decir, el patrón no es que a mayor edad el perfil cultural es más inactivo, sino todo lo contrario, dado que las personas de clase media mejoran su nivel de participación y amplían sus gustos a medida que aumenta su edad. Y si bien el comportamiento de esta variable no es completamente nítido, al menos sabemos que es muy diferente al que apuntábamos en los estudios anteriores. La edad, por tanto, no está necesariamente asociada a un ciclo de vida fatalista marcado

\footnotetext{
${ }^{21}$ Para la realización del presente análisis hemos empleado tablas que permiten estudiar con más precisión la relevancia de las categorías de cada una de las variables. Por razones de espacio, las hemos excluido del cuerpo del artículo. Por ello, de forma más sintética e intuitiva, hacemos referencia al gráfico 4 .
} 
por la biología, sino que su efecto tiene que ver con condiciones sociales ${ }^{22}$, cuyo entendimiento es clave para comprender las posibilidades de las personas, las cuales se enfrentan a un inevitable envejecimiento físico. En tercer lugar, hallamos una diferencia muy significativa entre la región metropolitana y el resto del país o lo que frecuentemente denominamos "regiones", teniendo la primera un perfil más participativo y cercano a la cultura más elevada, y mostrando las segundas un mayor grado de pasividad en los mismos términos.

Para finalizar, queremos destacar la asociación entre el eje 2 y la variable sexo. Esto es importante porque no siempre ha sido evidente que existe una fuerte cultura de género ${ }^{23}$. En un estudio anterior (Gayo et al., 2009), ya mencionado, no encontramos diferencias notorias. Sin embargo, combinando los hallazgos que hemos expuesto en los gráficos 3 y 4, podemos observar que está presente una divisoria cuya base se sostiene muy probablemente en diferencias de género. Por un lado, tendríamos una cultura masculina, la cual sobresale principalmente por su atención al deporte, y en menor medida por su gusto por el reggaetón y el hip-hop, junto con actividades como ir al zoológico y a parques de diversiones. Por otro lado, las mujeres se muestran más interesadas en la actualidad política nacional e internacional, las revistas de diseño y decoración, el cine de género dramático, la pintura y los museos de arte. En consecuencia, parece observarse una fractura entre dos culturas de género que podríamos expresar como dos conjuntos de elementos que se diferencian ostensiblemente: masculinidad/ deporte/ práctica/ corporalidad, por un lado, y femineidad/ conocimiento/ contemplación/ intelectualidad, por otro. Esto no significa que la alta cultura esté dominada por las mujeres, sino que hay subculturas de género que deben ser reconocidas como tales y que muy probablemente están vinculadas a lo que frecuentemente se entiende por ser hombre y mujer.

\footnotetext{
${ }^{22}$ Una investigación que avanza en esta línea de reflexión es la de Gayo (2012).

${ }^{23}$ Un estudio en el que se muestran diferencias de género relevantes es el de Bennett et al. (2009).
} 
Gráfico 4. Variables estructurantes del espacio de las prácticas culturales

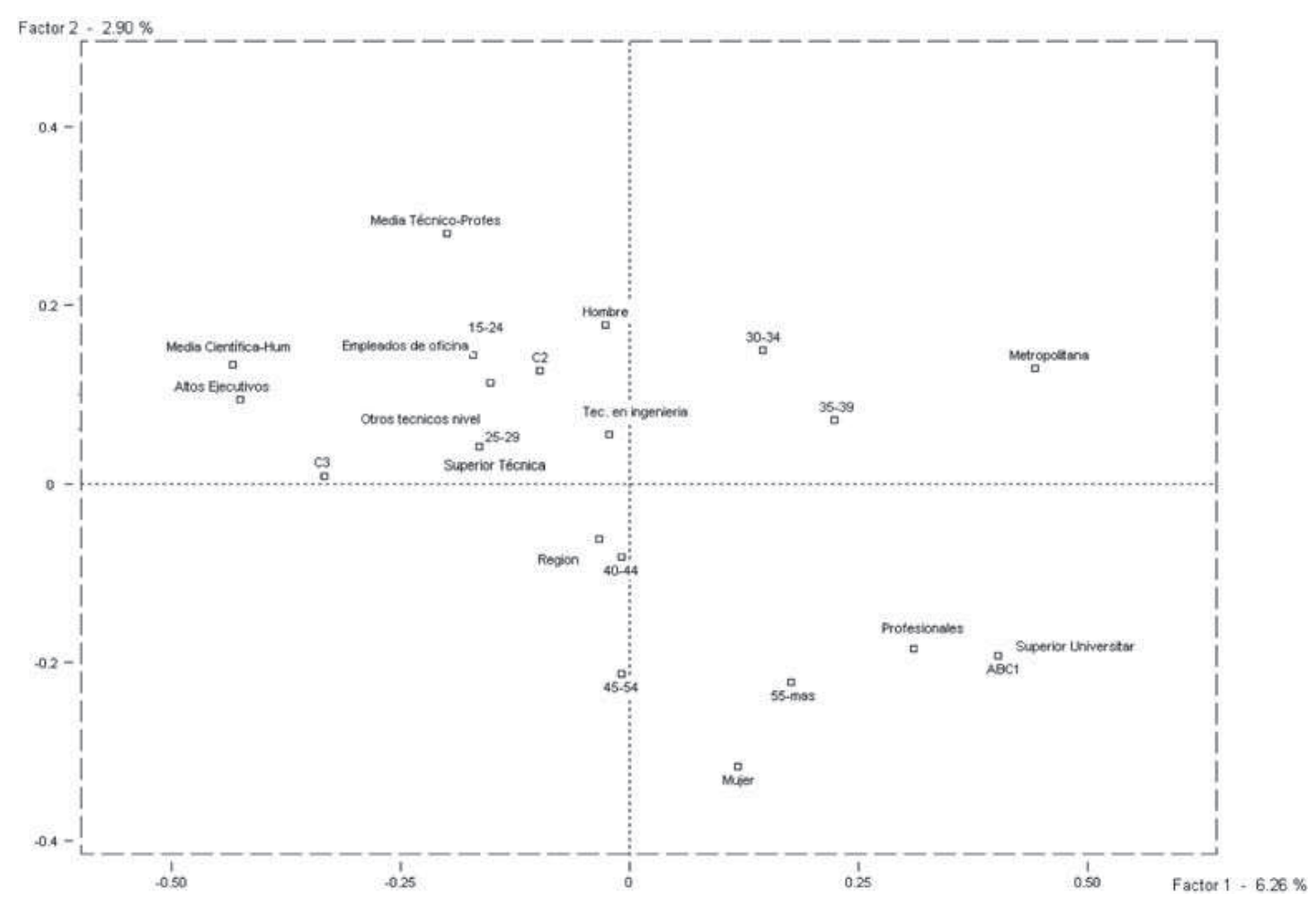

\section{CONCLUSIONES}

El espacio social de los estilos de vida en Chile se encuentra claramente estructurado de acuerdo a variables sociales cuya relevancia ha sido puesta en cuestión con particular fuerza en los últimas dos décadas por autores tan conocidos como Peterson, Beck, Baumann, entre otros. A este respecto es necesario subrayar con contundencia que la influencia de las condiciones socioeconómicas es un elemento esencial en la construcción de cualquier modelo explicativo completo de la participación y el gusto culturales. Así, independientemente de la visión que tengan los individuos de sí mismos, como más o menos individualizados, de las alteraciones a nivel del sistema, y por encima de cualquier modificación en la apertura de los grupos con mayores dotaciones de capitales económicos y culturales, es necesario destacar la importancia de las clases sociales y las desigualdades, esto último en un sentido más genérico. Desde un punto de vista ocupacional, esta división del espacio social chileno adopta una forma que podríamos calificar de "homología bi-polar" o polarizada en torno a dos grandes estilos de vida que 
encajan con dos conglomerados ocupacionales, a los que hemos denominado, siguiendo la nomenclatura clásica en la literatura sociológica, clase media y clase obrera. De este modo, estructura ocupacional y práctica cultural parecen encajar con notable precisión de una manera que difícilmente podemos decir que sea sorprendente. Los miembros de las ocupaciones de clase obrera se caracterizan por su bajo nivel de participación en actividades culturales, mientras los pertenecientes a la clase media se orientan con mucha mayor intensidad a la realización y disfrute de las mismas. Hablar de polos no significa que no haya individuos que presenten perfiles intermedios, sino que las ocupaciones se agrupan en torno a dos conjuntos que se constituyen como dos conglomerados que encajan en dos patrones de participación y gusto culturales bien diferentes. El de la clase media, participativo y vinculado a la alta cultura. El de la clase obrera, pasivo y asociado a la cultura popular y, a menudo, más masificada. Estos polos no son los extremos, sino dos modalidades posibles y distintas que nos hablan de diferencias ostensibles que muy probablemente los chilenos viven como tales. Esa polaridad nos habla de un campo de fuerzas que instituye al actuar de la clase media como un modelo normativo del que la mayoría está distante o carente.

El hecho de que la clase media sea participativa y muestre un relativamente elevado entusiasmo en identificarse con las actividades culturales ofrecidas en el cuestionario del CNCA, hace particularmente interesante su estudio. No en vano, los análisis de datos muestran que es el representante por antonomasia de la cultura con mayor legitimidad en la sociedad chilena actual. Sin embargo, en principio, ello no nos permite concluir nada sobre una eventual fragmentación interna, tema que ha estado en el centro de la reflexión sobre la clase media al menos durante los últimos treinta ańos. Es por esta razón que, enlazando con esta literatura, se hacía necesario explorar lo que sucedía en su interior. Efectivamente, se halló una importante heterogeneidad interna, pero ésta estaba lejos de oponer, por un lado, a los profesionales y ejecutivos, y, por otro, a los demás trabajadores, los que tendrían principalmente trabajos de oficina de menor nivel. Más bien, lo que se da es una oposición entre profesionales y las otras ocupaciones que conforman la clase media. En cualquier caso, sea como fuere la divisoria ocupacional, que es importante resaltar por haber sido la más común en las reflexiones sobre la clase media, es fundamental entender que esta clase, al igual que le sucedía al conjunto del espacio social, está estructurada principalmente de acuerdo con el nivel educativo. 
Éste está fuertemente asociado al tipo de ocupación y al grupo socioeconómico, pero en ningún caso es un reflejo de los mismos, sino más bien al contrario. Es por ello que, más allá de las tradicionales reflexiones sobre la importancia de la estructura ocupacional, es necesario hacer énfasis en la influencia de la educación en las prácticas culturales, lo que parece perfectamente comprensible, pues en términos bourdieuanos, ambos son tipos de capital cultural. Además, en contra de lo que nos sucedía para el conjunto del espacio social, el género aparece como un factor muy importante para entender las diferencias al interior de la clase media. Las diferentes inclinaciones relativas de hombres y mujeres permiten identificar un patrón de división que fragmenta a esta clase social en dos culturas. La masculina disfrutaría del deporte y las actividades al aire libre, y la femenina sería más sedentaria, intelectual y hogareńa. Esto podría estar dando señales sobre la reconfiguración de viejos roles, cuya transformación dejó probablemente casi intacto elementos como la mayor propensión a la presencia de los hombres en actividades fuera del hogar, y la preferencia de las mujeres por actividades cuya realización privilegia a menudo la ocupación del mismo.

\section{REFERENCIAS}

Araujo, Kathya y Martucelli, Danilo. "La inconsistencia posicional: un nuevo concepto sobre la estratificación social”, Revista CEPAL 103, 2011.

Ariztía, Tomás. "A New House in the Suburbs: Real Estate, Class and Culture in Santiago de Chile". Paper presentado en la conferencia "Re-Thinking Cultural Economy”, Reino Unido: Universidad de Manchester, 2007.

Barozet, Emmanuelle y Espinoza, Vicente. "¿Quiénes pertenecen a la clase media en Chile? Una aproximación metodológica”, Ecuador Debate 74 (2008): 103-122.

Beck, Ulrich y Beck-Gernsheim, Elisabeth. Individualization. London: Sage, 2001.

Bell, Daniel. The Coming of Post-Industrial Society. A Venture in Social Forecasting. London: Heinemann, 1974. 
Bennett, Tony; Emmison, Michael y Frow, John. Accounting for Tastes. Australian Everyday Cultures. Cambridge: Cambridge University Press, 1999.

Bennett, Tony; Savage, Mike; Silva, Elizabeth; Warde, Alan; Gayo-Cal, Modesto y Wright, David."Cultural formations of the middle clases". En Culture, Class, Distinction. London: Routledge (2009): 177-194.

Bernstein, Basil. Clases, códigos y control. I. Estudios teóricos para una sociología del lenguaje. Madrid: Akal, 1989.

Bonham, John. The Middle Class Vote. London: Faber and Faber, 1954.

Bourdieu, Pierre. La distinction. Paris: les Éditions de Minuit, 1979.

La noblesse d'état. Grandes écoles et esprit de corps. Paris: Les Éditions de Minuit, 1989.

----------. Las reglas del arte. Génesis y estructura del campo literario. Barcelona: Anagrama, 2005.

Brooks, Clen y Manza, Jeff. "Partisan Alignments of the 'Old' and 'New' Middle Classes in Post-Industrial America”. En Terry Nichols Clark y Michel Rempel (eds.). Citizen politics in post-industrial Societies. London: Westview Press (1997): 143-157.

Candina, Azun. Por una vida digna y decorosa: Clase media y empleados públicos en el siglo XX chileno. Santiago de Chile: Facultad de Filosofía y Humanidades, Universidad de Chile, 2009.

Castells, Manuel. The Information Age: Economy, Society, and Culture. Oxford (Reino Unido) y Cambridge (Massachussets, EE.UU.), Blackwell Publishers, 2003.

Chauvel, Louis. Les classes moyennes à la dérive. Éditions du Seuil et La République des Idées, 2006. 
Consejo Nacional de la Cultura y las Artes. Segunda Encuesta Nacional de Participación y Consumo Cultural. Valparaíso: Ediciones Cultura, 2011.

Devine, Fiona. Class Practices. How Parents Help Their Children Get Good Jobs. Cambridge: Cambridge University Press, 2004.

Erikson, Robert y Goldthorpe, John H. The Constant Flux, A Study of Class Mobility in Industrial Societies. Oxford: Clarendon Press, 1993.

Filgueira, Carlos y Geneletti, Carlo. Estratificación y Movilidad Ocupacional en América Latina. Santiago de Chile: CEPAL, 1981.

Franco, Rolando; León, Arturo y Atria, Raúl (eds.). Estratificación y movilidad social en América Latina. Transformaciones estructurales de un cuarto de siglo. Santiago de Chile: LOM, CEPAL and GTZ, 2007.

Franco, Rolando, Hopenhayn, Martín y León, Arturo (coords.). Las clases medias en América Latina. Retrospectiva y nuevas tendencias. México: CEPAL, Siglo XXI Editores, 2011.

Gayo, Modesto. "Leisure and participation in Britain", Cultural Trends 58/59, volume 15, issue 2/3, June-September (2006): 175-192.

"La desigualdad socioeconómica y el gusto musical en Chile". En Politicas Culturales: Contingencia y Desafios. Colección del Observatorio de Políticas Culturales (OPC) N¹, Año 1. Santiago de Chile: LOM Ediciones (2011): 75-85.

"Del tango al hip-hop: el cambio de los gustos musicales en Chile". En Politicas Culturales: ¿Qué medimos? ¿Cómo evaluamos? Colección del Observatorio de Políticas Culturales (OPC) №2, Ańo 2. Santiago de Chile: LOM Ediciones (2012): 55-65.

Gayo-Cal, Modesto; Savage, Mike y Warde, Alan. "A Cultural Map of the United Kingdom 2003”, Cultural Trends 15/2-3 (2006): 213-237. 
Gayo, Modesto; Teitelboim, Berta y Méndez, María Luisa. "Patrones culturales de uso del tiempo libre en Chile. Una aproximación desde la teoría bourdieuana”, Universum 24/2 (2009): 42-72.

Gayo, Modesto y Teitelboim, Berta. "Localismo, cosmopolitismo y gustos musicales”. En Reporte Encuesta Nacional de Opinión Pública de la Universidad Diego Portales (4a versión): Radiografía social, politica y económica de Chile. Santiago de Chile, año 2, no 2, abril (2009a): 111-120.

"La producción social del capital cultural: ¿privilegio o mérito?”. En Chile 2009: percepciones y actitudes sociales. Informe de la Quinta Encuesta Nacional UDP. Informe anual de la sexta encuesta nacional UDP. Santiago: ICSO-UDP (2009b): 85-94.

Gayo, Modesto (director); Méndez, María Luisa; Radakovich, Rosario y Wortman, Ana. Consumo cultural y desigualdad de clase, género y edad: un estudio comparado en Argentina, Chile y Uruguay. Madrid: Fundación Carolina, Serie Avances de Investigación 62, 2011.

Heath, Anthony y Savage, Mike. "Middle-class politics". En Roger Jowell et al. (eds.). British Social Attitudes: the $11^{\text {th }}$ report. Aldershot: Darmouth Publishing (1994): 61-73.

Kriesi, Hanspeter. "New social movements and the new class in the Netherlands", American Journal of Sociology 94/5 (1989): 1078-1116.

Kuper, Adam. Cultura. La versión de los antropólogos. Barcelona: Paidós (1a edición en inglés, 1999), 2001.

Lamont, Michele. Money, Morals and Manners: The Culture of the French and American Upper-Middle Class. Chicago: The University of Chicago Press, 1992.

León, Arturo y Martínez, Javier. La estratificación social chilena hacia fines del siglo XX. Santiago de Chile: CEPAL, Serie 52, 2001. 
Lipset, Seymur M. El hombre politico. Las bases sociales de la política. Madrid: Editorial Tecnos, 1987.

Martínez, Javier y Tironi, Eugenio. Las Clases Sociales en Chile: cambio y estratificación, 1970-1980. Santiago de Chile: Ediciones Sur, 1985.

Méndez, María Luisa. "Experiencias y Significados asociados a la Movilidad Social”, Revista del Magister en Antropología y Desarrollo. Antropología Social Aplicada 6, 2003.

"Do you see $\mathrm{m}$ as I see myself?: Middle class identities in Chile". Ph. D thesis. No publicada. United Kingdom: University of Manchester, 2007.

"Middle Class Identities in a Neoliberal age: tensions between contested authenticities", The Sociological Review 56/2 (2008): 220-237.

Méndez, María Luisa y Gayo, Modesto. "El perfil de un debate: movilidad y meritocracia. Contribución al estudio de las sociedades latinoamericanas". En Rolando Franco; Arturo León y Raúl Atria (coords.). Estratificación y movilidad social en América Latina. Transformaciones estructurales de un cuarto de siglo. Santiago de Chile: LOM ediciones (2007): 121-157.

Méndez, María Luisa y Barozet, Emmanuelle. "Lo auténtico también es público. Comprensión de lo público desde las clases medias en Chile", Polis, Revista Latinoamericana 11/31 (2012): 183-202.

Mills, Charles Wright. White Collar. The American Middle Classes. New York: Oxford University Press, 1959.

Peterson, Richard A. y Kern, Roger M. "Changing highbrow taste: from snob to omnivore”, American Sociological Review 61/5 (1996): 900-907.

Roberts, Ken. Leisure. London: Longman, 1970. 
"Leisure Inequalities, Class Divisions and Social Exclusion in Present-day Britain”, Cultural Trends 13/2 (2004): 57-71.

Rootes, Christopher. "A New Class? The Higher Educated and the New Politics". En Luis Maheu (ed.). Social Movements and Social Classes: The Future of Collective Action. London: SAGE (1995): 220-235.

Salazar, Gabriel y Pinto, Julio. Historia contemporánea de Chile. Volúmenes I al V. Santiago de Chile: Ediciones LOM, 1999-2002.

Savage, Mike; Barlow, James; Dickens, Peter y Fielding, Tony. Property, Bureaucracy and Culture. Middle-Class Formation in Contemporary Britain. London: Routledge, 1995.

Sémbler, Camilo. Estratificación social y clases sociales. Una revisión analitica de los sectores medios. Santiago de Chile: CEPAL, Serie Políticas Sociales 125, 2006.

Torche, Florencia. "Unequal But Fluid: Social Mobility in Chile in Comparative Perspective”, American Sociological Review 70/3 (2005): 422-450.

Torche, Florencia y Wormald, Guillermo. Estratificación y movilidad social en Chile: entre la adscripción y el logro". Santiago de Chile: CEPAL, Serie Políticas Sociales 98, 2004. 


\section{Anexo 1.}

Tabla 1. Ocupaciones codificadas en base a la Clasificación Internacional Uniforme de Ocupaciones de 1988 de la OIT. En negrita los grupos ocupacionales de la clase media, y en cursiva los de la clase obrera.

\begin{tabular}{|c|c|c|}
\hline & Cód. & Categorías \\
\hline Altos ejecutivos & $\begin{array}{l}112 \\
121 \\
122 \\
123\end{array}$ & $\begin{array}{l}\text { Personal Directivo administración publica } \\
\text { Directores generales y gerentes generales de empresas } \\
\text { Directores departamentos de producción y operaciones } \\
\text { Otros directores de departamentos }\end{array}$ \\
\hline Profesionales & $\begin{array}{l}213 \\
214 \\
221 \\
222 \\
223 \\
231 \\
232 \\
233 \\
234 \\
235 \\
241 \\
242 \\
244 \\
245 \\
246\end{array}$ & $\begin{array}{l}\text { Profesionales de la informática } \\
\text { Arquitectos, ingenieros y afines } \\
\text { Prof. en ciencias biológicas } \\
\text { Médicos y profesionales afines } \\
\text { Personal de enfermería y partería de nivel superior } \\
\text { Profesores de establecimientos de la enseñanza superior } \\
\text { Profesores de enseñanza secundaria } \\
\text { Maestros nivel superior enseñanza primaria y preescolar } \\
\text { Maestros de nivel superior enseñanza especial } \\
\text { Otros profesionales de la enseñanza } \\
\text { Especialista en organización y administración de empresas } \\
\text { Profesionales del derecho } \\
\text { Especialista en ciencias sociales y humanas } \\
\text { Escritores, artistas creativos y ejecutantes } \\
\text { Sacerdotes de distintas religiones }\end{array}$ \\
\hline $\begin{array}{l}\text { Técnicos de la } \\
\text { ingeniería }\end{array}$ & $\begin{array}{l}311 \\
312 \\
313 \\
314 \\
315 \\
321 \\
322 \\
323 \\
324 \\
\end{array}$ & $\begin{array}{l}\text { Técnico ciencias físicas y químicas e ingeniería } \\
\text { Técnico en programación y control informáticos } \\
\text { Operadores de equipos ópticos y electrónicos } \\
\text { Técnicos en navegación marítima y aeronáutica } \\
\text { Insp.de obras, seguridad, salud y control de calidad } \\
\text { Técnico nivel medio ciencias biológicas } \\
\text { Prof. nivel medio de la salud } \\
\text { Personal de enfermería y partería de nivel medio } \\
\text { Practicantes de medicina tradicional y curanderos }\end{array}$ \\
\hline Profesores básicos & $\begin{array}{l}332 \\
333 \\
\end{array}$ & $\begin{array}{l}\text { Maestros de nivel medio de la enseñanza preescolar } \\
\text { Maestros de nivel medio de la enseñanza especial }\end{array}$ \\
\hline $\begin{array}{l}\text { Otros técnicos nivel } \\
\text { medio }\end{array}$ & $\begin{array}{l}341 \\
342 \\
343 \\
344 \\
346 \\
347\end{array}$ & $\begin{array}{l}\text { Prof. nivel medio en operaciones financieras y comerciales } \\
\text { Agentes comerciales y corredores } \\
\text { Prof.de nivel medio de servicios de administrativos } \\
\text { Agentes de administración pública de aduanas, impuestos } \\
\text { Trabajadores y asistentes sociales de nivel medio } \\
\text { Prof. de nivel medio de actividades artísticas y deportes }\end{array}$ \\
\hline
\end{tabular}




\begin{tabular}{|c|c|c|}
\hline Empleados de oficina & $\begin{array}{l}411 \\
412 \\
413 \\
414 \\
419 \\
\end{array}$ & $\begin{array}{l}\text { Secretarios y operarios de máquinas de oficina } \\
\text { Auxiliares contables o financieros } \\
\text { Empleados encargados reg.de materiales y transportes } \\
\text { Empleados bibliotecas y servicios de correos } \\
\text { Otros oficinistas }\end{array}$ \\
\hline $\begin{array}{l}\text { Empleado que atiende } \\
\text { clientes }\end{array}$ & $\begin{array}{l}421 \\
422 \\
\end{array}$ & $\begin{array}{l}\text { Cajeros, taquilleros y afines } \\
\text { Empleados de servicios de información a la clientela }\end{array}$ \\
\hline $\begin{array}{l}\text { Trab. de los ser. } \\
\text { Personales }\end{array}$ & $\begin{array}{l}511 \\
512 \\
513 \\
514 \\
515 \\
516\end{array}$ & $\begin{array}{l}\text { Personal al servicio directo de los pasajeros } \\
\text { Personal de intendencia y de restauración } \\
\text { Trabajadores de los cuidados personales y afines } \\
\text { Otros trabajadores de servicios personales a particulares } \\
\text { Astrólogos, adivinadores y afines } \\
\text { Personal de los servicios de protección y seguridad }\end{array}$ \\
\hline Empleados de comercio & $\begin{array}{l}522 \\
523 \\
\end{array}$ & $\begin{array}{l}\text { Vendedores y demostradores de tiendas } \\
\text { Vendedores de quioscos y puestos de mercado }\end{array}$ \\
\hline $\begin{array}{l}\text { Trabajadores calificados } \\
\text { de agro y pesca }\end{array}$ & $\begin{array}{l}611 \\
612 \\
614 \\
615 \\
\end{array}$ & $\begin{array}{l}\text { Agricultores y trabajadores calificados de cultivos para el mercado } \\
\text { Criadores y trabajadores pecuarios calificados } \\
\text { Trabajadores forestales calificados y afines } \\
\text { Pescadores, cazadores y tramperos }\end{array}$ \\
\hline $\begin{array}{l}\text { Operario de la ind. de } \\
\text { extracción y construcción }\end{array}$ & $\begin{array}{l}711 \\
712 \\
713 \\
714 \\
\end{array}$ & $\begin{array}{l}\text { Mineros, canteros, pegadores y labrantes de piedra } \\
\text { Operarios de la construcción (obra gruesa) } \\
\text { operarios de la construcción Obra terminada } \\
\text { Operarios de la industria construcción y extracción }\end{array}$ \\
\hline $\begin{array}{l}\text { Operario de la ind. } \\
\text { metalúrgica y mecánica }\end{array}$ & $\begin{array}{l}721 \\
722 \\
723 \\
724 \\
\end{array}$ & $\begin{array}{l}\text { Moldeadores, soldadores, chapistas, caldereros } \\
\text { Herreros, herramientas y afines } \\
\text { Mecánicos y ajustadores de m quinas } \\
\text { Mecánicos y ajustadores de equipos eléctricos }\end{array}$ \\
\hline $\begin{array}{l}\text { Mecánicos de precisión y } \\
\text { artesanos }\end{array}$ & $\begin{array}{l}731 \\
732 \\
733 \\
734 \\
741 \\
742 \\
743 \\
744\end{array}$ & $\begin{array}{l}\text { Mecánicos de precisión en metales y materiales similares } \\
\text { Alfareros, operarios de cristalerías y afines } \\
\text { Artesanos de madera, tejidos cuero y materiales similares } \\
\text { Operarios de las artes gráficas y afines } \\
\text { Operarios del procesamiento de alimentos y afines } \\
\text { Operarios del tratamiento de madera, ebanistas y afines } \\
\text { Operarios de los textiles y la confección y afines } \\
\text { Operarios de las pieles, cuero y calzado }\end{array}$ \\
\hline
\end{tabular}




\begin{tabular}{|c|c|c|}
\hline \multirow{14}{*}{ Operarios de maquinaria } & 811 & $\begin{array}{l}\text { Operadores de instalaciones mineras, extracción y proces.de } \\
\text { minerales }\end{array}$ \\
\hline & 812 & Operadores de instalaciones de procesamiento de metales \\
\hline & 813 & Operadores de instalaciones de vidriería, cerámica y afines \\
\hline & 814 & $\begin{array}{l}\text { Operadores de instalaciones de proceso de madera y de la } \\
\text { fab.de papel }\end{array}$ \\
\hline & 815 & Operadores de instalaciones de tratamientos químicos \\
\hline & 816 & Operadores de instalaciones de producción de energía y \\
\hline & 822 & Operadores de máquinas para fabricar productos químicos \\
\hline & 823 & $\begin{array}{l}\text { Operadores de maquinaria para fabricar prod.de caucho y } \\
\text { material plástico }\end{array}$ \\
\hline & 824 & Operadores de máquinas para fabricar productos de madera \\
\hline & 825 & $\begin{array}{l}\text { Operadores de maquinaria de imprenta, encuadernación y } \\
\text { fabrica producción de papel }\end{array}$ \\
\hline & 826 & $\begin{array}{l}\text { Operadores de maquinaria para fabricar productos textiles y } \\
\text { artículos de piel y cuero }\end{array}$ \\
\hline & 827 & $\begin{array}{l}\text { Operadores de maquinaria para elaborar alimentos y } \\
\text { productos afines }\end{array}$ \\
\hline & 828 & Montadores \\
\hline & 829 & Otros operadores de $m$ guinas y montadores \\
\hline \multirow{4}{*}{ Conductores } & 831 & Maquinistas de locomotoras y afines \\
\hline & 832 & Conductores de vehículos de motor \\
\hline & 833 & $\begin{array}{l}\text { Operadores de maquinaria agrícola y otras maquinarias } \\
\text { móviles }\end{array}$ \\
\hline & 834 & Marineros de cubierta y afines \\
\hline \multirow{6}{*}{$\begin{array}{l}\text { Trabajadores no } \\
\text { calificados de ventas y } \\
\text { servicios }\end{array}$} & 911 & Vendedores ambulantes y afines \\
\hline & 912 & Limpiabotas y otros trabajadores callejeros \\
\hline & 913 & Personal domestico y afines \\
\hline & 914 & Conserjes, lavadores de ventanas y afines \\
\hline & 915 & Mensajeros, porteros y afines \\
\hline & 916 & Recolectores de basura y afines \\
\hline \multirow{4}{*}{$\begin{array}{l}\text { Peones sector primario y } \\
\text { secundario }\end{array}$} & 921 & Peones agropecuarios, forestales, pesqueros y afines \\
\hline & 931 & Peones de la minería y la construcción \\
\hline & $\frac{932}{020}$ & Peones de la industria manufacturera \\
\hline & 933 & Peones del transporte \\
\hline
\end{tabular}


Tabla 2. Grupos ocupacionales dentro de las clases media y obrera y sus frecuencias.

\begin{tabular}{|l|c|c|c|}
\hline & Clase media & Clase obrera & Total \\
\hline Altos Ejecutivos & 32 & 0 & 32 \\
\hline Profesionales & 225 & 0 & 225 \\
\hline Técnico en ingeniería & 56 & 0 & 56 \\
\hline Profesores Básicos & 0 & 9 & 9 \\
\hline Otros técnicos nivel medio & 120 & 0 & 120 \\
\hline Empleados de oficina & 109 & 0 & 109 \\
\hline Empleados que atienden clientes & 0 & 58 & 58 \\
\hline $\begin{array}{l}\text { Trabajadores de los servicios } \\
\text { personales }\end{array}$ & 0 & 142 & 142 \\
\hline Empleados de comercio & 0 & 269 & 269 \\
\hline Trabajadores calif. & 0 & 22 & 22 \\
\hline $\begin{array}{l}\text { Operario de industria extracción } \\
\text { construcción }\end{array}$ & 0 & 148 & 148 \\
\hline $\begin{array}{l}\text { Operario de ind. metalúrgica y } \\
\text { mecánica }\end{array}$ & 0 & 91 & 91 \\
\hline $\begin{array}{l}\text { Mecánicos de precision y } \\
\text { artesanos }\end{array}$ & 0 & 116 & 116 \\
\hline Oper.de maquinaria & 0 & 49 & 49 \\
\hline Conductores & 0 & 149 & 149 \\
\hline $\begin{array}{l}\text { Trabajadores no calificados de } \\
\text { ventas y servicios }\end{array}$ & 0 & 174 & 174 \\
\hline $\begin{array}{l}\text { Peones sector primario y } \\
\text { secundario }\end{array}$ & 0 & 150 & 150 \\
\hline Ocupado s/o & $\mathbf{2 7 , 8 \%}$ & $72,2 \%$ & $\mathbf{1 0 0 , 0 \%}$ \\
\hline Total & & 33 & 33 \\
\hline \% & 0 & 1410 & 1952 \\
\hline
\end{tabular}

